\title{
Saillance inhérente et saillance discursive en movima
}

\author{
Katharina Haude*
}

\section{INTRODUCTION}

La notion «saillance» peut être vue de deux perspectives : d'un côté, la " hiérarchie d'animacité » (animacy hierarchy; Comrie 1989, Croft 2003, inter alia), avec toutes ses sous-catégories, peut se caractériser en termes de saillance, car une entité haute dans cette hiérarchie (humaine, connue, présente dans la conversation) est plus encline à capter et retenir l'attention d'un humain qu'une entité basse dans cette hiérarchie (voir la notion " ontological salience » dans Klaiman 1991: 162); nous allons appeler ce type de saillance «saillance inhérente », car elle renvoie à une propriété d'une expression nominale. D'un autre côté, toutes les langues offrent des stratégies discursives, par exemple au moyen de variations dans l'ordre des mots, avec lesquelles le locuteur peut diriger l'attention de l'interlocuteur vers une entité ; nous appelons cette mise en saillance induite dans le discours "saillance discursive ». Ces deux côtés de la notion ne sont pas forcément reliés. Or, il y a des langues où la saillance inhérente a un effet direct sur la morphosyntaxe, et dans ces langues on peut s'attendre à ce que cela influence aussi les stratégies syntaxiques de saillance discursive. Le movima (isolat, Amazonie bolivienne ; Haude 2006) est une langue de ce type.

La syntaxe du movima est fortement configurationnelle: le noyau d'une proposition transitive est à prédicat initial, avec un argument interne et un argument externe au syntagme prédicatif ${ }^{1}$. L'argument interne renvoie au participant supérieur dans les hiérarchies de personne $(1>2>3)$ et de topicalité $(3$ topique $>3$ non topique), l'argument externe renvoie au participant inférieur dans ces hiérarchies. Cet article explore l'interaction de cette grammaticalisation de saillance inhérente avec la mise en saillance d'un participant par un pronom libre à l'extérieur du noyau, au début de la proposition. Nous allons montrer que non seulement selon sa fonction pragmatique (focalisation ou topique resomptif), mais aussi selon le statut syntaxique de l'argument, qui est déterminé par la saillance inhérente de son référent, la construction à pronom initial prend des formes différentes : a) lorsque le pronom libre renvoie au participant inférieur

* SeDyL (UMR 8202), CNRS, INaLCO, IRD; haude@vjf.cnrs.fr

1 Je prends le terme noyau comme équivalent de "core» de l'anglais (prédicat + argument(s)). Les termes "argument interne » et " argument externe », introduits par Williams (1981), sont appliqués ici pour faire référence aux positions syntaxiques des syntagmes en termes de constituance, indépendamment de leur rôles sémantiques. 
dans la hiérarchie, la structure du noyau n'est pas affectée ; b) lorsque le pronom libre renvoie au participant supérieur dans la hiérarchie, une opération détransitivisante prend place, car l'argument interne ne peut pas être extrait du syntagme prédicatif pour être focalisé. De cette façon, la saillance inhérente des participants a un effet direct sur la stratégie syntaxique de mise en saillance discursive. Cependant, dans certains cas l'argument interne peut sortir du noyau et laisser sa place à l'ancien argument externe, contredisant la hiérarchie de saillance inhérente, ce qui montre que la saillance discursive peut être privilégiée par rapport à la saillance inhérente.

L'article est structuré comme suit. La section 2 décrit la structure de la proposition transitive. La section 3 présente l'effet de la hiérarchie de personne $(1>2>3)$ sur le codage des arguments. La section 4 présente une construction syntaxique qui permet de rendre saillante une troisième personne dans un scénario $3>3$. La section 5 montre comment cette même construction est appliquée avec des pronoms interlocutifs (première et deuxième personne), et qu'ici, des fins discursives peuvent dépasser les facteurs de saillance inhérente. La section 6 conclut que même si, à priori, la saillance inhérente d'un référent détermine la structure de la proposition, elle peut être neutralisée à des fins discursives.

Les données proviennent d'un corpus de transcriptions d'environ 20 heures de textes oraux de différents genres (narratif, descriptif, dialogue etc.) ${ }^{2}$.

\section{STRUCTURE DE LA PROPOSITION TRANSITIVE}

La structure du noyau transitif movima est présentée en (1) : il est composé du syntagme prédicatif qui contient un premier argument, que j'appelle argument interne, et un deuxième argument, que j'appelle argument externe (voir note 1).

(1) [prédicat=argument] [argument]

Les arguments du noyau sont identifiés par l'absence du marqueur oblique, $n$-. Leur différence de constituance (interne ou externe au syntagme prédicatif) apparaît dans les propriétés suivantes du pronom ou syntagme nominal (SN) qui l'exprime :

2 Le corpus de textes a été assemblé pendant les projets «Lexion and Syntax » à la Radboud Universiteit Nijmegen (2001-2005), le projet de documentation du movima (DoBeS, Volkswagenstiftung Az. II/81914, 2006-2011), et le projet EuroBABEL-RHIM (DFG Ha/5910/1-1). Les données sont intégrées dans l'archive DoBeS (www.mpi.nl/DOBES). Son contenu fut présenté au séminaire «Typology of information structure » au Max-Planck-Institut für Psycholinguistik, Nijmegen, en décembre 2010. Je remercie les locuteurs du movima de m'avoir fourni les données, le public de la présentation pour les remarques, quatre membres anonymes du comité de lecture pour leurs commentaires sur la première version de cet article et à Francesc Queixalós, Odile Lescure et Annie Montaut pour la correction du français. Pour plus d'information sur la grammaire movima, voir Haude (2006). 
- position : $\quad$ interne, directement après le prédicat ;

externe, après l'argument interne, parfois avec d'autres éléments (particules) intermédiaires ;

- conditions d'expression : interne obligatoire (zéro $=$ ' $1 \mathrm{SG}^{\prime}$ '); externe non obligatoire si connu du contexte ;

- cliticisation: interne, toujours cliticisé $($ symbole $=)($ effet : nouveau mot prosodique);

externe seulement cliticisé quand pronom (symbole -- ; seul effet : resyllabification avec une consonne précédente)

Les rôles sémantiques (agent/patient) sont indiqués par des morphèmes verbaux, le marqueur « direct » indiquant que l'argument interne est l'agent et l'externe le patient, le marqueur « inverse » indiquant la configuration inverse. Le contraste direct/inverse est illustré en (2) et (3), respectivement, avec un pronom de $2^{\mathrm{e}}$ personne $(=n)$ et un syntagme nominal $(\mathrm{SN})$ représentant une $3^{\mathrm{e}}$ personne. Le marquage direct en (2) indique que la $2^{\mathrm{e}}$ personne est l'agent et la $3^{\mathrm{e}}$ le patient, le marquage inverse en (3) indique que la $2^{\mathrm{e}}$ est le patient et la $3^{\mathrm{e}}$ l'agent.

(2)

$$
\begin{aligned}
& \text { joy-a- } t e=n \quad \text { as weykawa:yo } \\
& \text { aller-DR-CO=2 ART.N bœuf_pour_monter } \\
& \text { 'Tu emmènes le bœuf pour monter' }
\end{aligned}
$$

$$
\begin{array}{lll}
\text { joy-te- } \boldsymbol{k a y}-\boldsymbol{a}=n & \text { us } & \text { Gerardo } \\
\text { aller-CO-INV-EP=2 } & \text { ART.M } & \text { Gerardo }
\end{array}
$$

\section{LES HIERARCHIES DES PERSONNES ET DE TOPICALITE}

La hiérarchie des personnes en movima est $1>2>3$. Les personnes locales $\left(1^{\mathrm{e}}\right.$ et $2^{\mathrm{e}}$ ) ne peuvent être exprimées dans le noyau transitif que par l'argument interne. Lorsque les deux interagissent, c'est seulement la $1^{\mathrm{e}}$ qui est marquée sur le verbe (zéro), la $2^{\mathrm{e}}$ se comprend par le contexte ou est exprimée par un pronom libre (voir section 5). (Seule la $2^{\mathrm{e}}$ personne du pluriel peut être exprimée comme argument externe, dans le scénario où elle interagit avec une $1^{\mathrm{e}}$ personne; voir Haude 2011).

$$
\begin{aligned}
& \text { jayna rey it man }<a:>y e=\varnothing \\
& \text { DSC MOD } 1 \text { rencontrer }<\text { DR }>=1 \mathrm{SG} \\
& \text { 'Je (te) retrouverai.' }
\end{aligned}
$$

${ }^{3} \mathrm{La} 1^{\mathrm{e}}$ personne est optionnellement exprimée par un élément proclitique (ici, $\left.i t\right)$ dans le noyau, en plus de l'enclitique (Haude 2011). Cet élément n'est pas identique au pronom libre et n'a aucun effet pragmatique. 
$\begin{array}{clll}\text { (5) it manne: } \boldsymbol{k} \boldsymbol{k a y}=\varnothing & \text { di:ran } & n \text { - } a s & j i<w a \sim>w a=n \\ 1 \text { rencontrer-INV=1SG } & \text { encore } & \text { OBL-ART.N } & \text { venir }<\mathrm{NML}>=2\end{array}$

'(Tu) me retrouveras encore quand tu viendras.'

Pour désigner un événement comportant une personne locale $\left(1^{\mathrm{e}}\right.$ ou $2^{\mathrm{e}}$ personne $)$ et une $3^{\mathrm{e}}$ personne, il n'y a pas de choix pour le codage des arguments dans le noyau syntaxique : la personne locale est toujours l'argument interne et la $3^{\mathrm{e}}$ personne l'argument externe. Cela a été illustré en (2) et (3) ci-dessus.

Par contraste avec la $1^{\mathrm{e}}$ et $2^{\mathrm{e}}$ personnes, quand les deux participants d'un événement transitif sont des $3^{\text {es }}$ personnes, il n'y a pas de restriction formelle pour leur expression comme argument interne ou externe : une $3^{\mathrm{e}}$ personne peut être exprimée par un pronom lié ou un syntagme nominal ( $\mathrm{SN}$ ) en position aussi bien interne qu'externe. Or, l'argument interne est presque toujours exprimé par un pronom, le cas où il est un SN étant exceptionnel, tandis que l'argument externe est souvent SN. Une analyse d'un texte narratif d'environ 120 propositions a montré que les participants principaux sont introduits par des SNs dans des propositions intransitives (en accord avec DuBois 1987), pour, après, être présentés, dans des propositions transitives, par des pronoms en position interne. Le facteur le plus important pour le choix de la position syntagmatique est donc le statut discursif (topicalité). La priorité du pronom en position interne, qui conditionne le choix de la forme verbale directe ou inverse, est illustré dans l'exemple (6) : le pronom est en position interne, et le verbe est direct là où le pronom représente l'agent et inverse là où il représente le patient.

$$
\begin{aligned}
& \text { asko ona-waj-na='ne chot i'ne [...] } \\
& \text { PRO. } 3 \mathrm{~N} . \mathrm{AB}^{4} \text { connaître-CL.lieu-DR }=3 \mathrm{~F} \text { toujours PRO.3F } \\
& \text { joy-te-kay-a='ne i'nes a:kay-a='ne } \\
& \text { aller-CO-INV-EP }=3 \mathrm{~F} \quad \text { ART.F frère/sœur aîné-EP }=3 \mathrm{~F} \\
& \text { 'Elle connaissait ce (lieu)-là, elle. Sa sœur âinée l'avait toujours emmenée.' }
\end{aligned}
$$

Quand les deux participants dans un événement transitif sont exprimés de la même façon, on trouve, à très peu d'exceptions, la construction directe (voir aussi Haude 2010). En (7), les deux participants sont exprimés par des SNs (=as mimi:di est l'argument interne en (7)) ; en (8), ils sont exprimés par des pronoms liés ( $=i s$ est 1'argument interne ici). ${ }^{5}$ Le fait que la construction directe est préférée ici, montre qu'en cas d'égalité d'expression, qui réflète une égalité du statut discursif, l'agent est conçu comme le plus saillant. En revanche, l'exemple (7) montre que des facteurs comme humanitude et proximité émotionnelle du locuteur, propriétés du fils dans cet exemple, ne sont pas des facteurs primaires pour le choix de l'argument interne.

\footnotetext{
${ }^{4} \mathrm{La}$ glose 'AB' indique que le pronom ou article renvoie à une entité absente; la glose 'PSE' indique qu'un article renvoie à une entité qui n'existe plus (voir Haude 2006). Ces catégories ne sont pas inclues dans les traductions pour des raisons d'espace.

5 Le marqueur $-k$ - 'OBV' devant le pronom externe apparaît seulement quand l'argument interne est de ou contient une $3^{\mathrm{e}}$ personne.
} 
(7)

$\begin{array}{lll}\text { jay, } & \text { jayna } & \text { lap-na=as mimi:di us majni } \\ \text { EXCL } & \text { DSC } & \text { mordre-DR=ART.N serpent ART.M }\end{array}$

mon enfant

'Ah, maintenant un serpent a mordu mon fils !'

(8) tap-na=is-k-i'ne, bot-'oj-et-na=is,

baigner-DR=3PL.AB--OBV-3F changer-CL. vêtements-APPL-DR=3PL.AB

joro-poj-na=is-k-i'ne

dormir-CAUS-DR $=3$ PL.AB-- OBV-3F

'Ils la baignèrent, ils (la) changèrent, ils la couchèrent.'

La construction inverse, de son côté, contient en général un pronom comme argument interne et un SN comme argument externe, comme on pouvait l'observer en (6).

\section{SAILLANCE DISCURSIVE : TROISIEMES PERSONNES}

Un participant peut être exprimé par un pronom libre (PRO) devant le prédicat, sans rupture prosodique (il n'y a pas de pause). Cette construction (appelée « marked-topic construction » dans des publications précédentes, Haude 2009), sert comme stratégie pour rendre saillant ce participant. Ici, nous allons nous concentrer sur la fonction où le pronom libre renvoie à un nouveau participant introduit immédiatement auparavant ${ }^{6}$; une autre fonction, celle de reprise d'un ancien topique du discours, sera illustrée brièvement. En fonction du participant mis en relief, il y a deux variantes de cette construction, qui seront décrites cidessous :

a) PRO correspond à l'argument externe : pas d'effet sur le noyau

b) PRO correspond à l'argument interne : opération détransitivisante

\section{a) PRO correspond à l'argument externe}

Le participant exprimé par le pronom en position initiale dans cette construction est, en termes de saillance inhérente, inférieur au participant codé comme argument interne. Comme d'habitude, le verbe est marqué comme direct (9) ou inverse (10) selon le rôle sémantique de l'argument interne. Le pronom libre a donc un statut identique à celui de l'argument externe, qu'il remplace (il y a cependant quelques exemples où l'argument externe est en outre exprimé par un $\mathrm{SN})$. Le pronom reprend le participant introduit immédiatement auparavant, comme le montrent les exemples.

6 Pour cet article il suffira de parler d'une "mise en saillance » pour caractériser cette construction. Nous ne pouvons à ce moment-ci offrir une définition exacte de la fonction de cette construction, car cela demanderait une discussion à part. Elle est peut-être plus proche d'une focalisation (et en accord avec cela, est souvent traduite par une clivée), parfois signalant une rupture dans la chaîne thématique, parfois un contraste avec d'autres référents possibles. 
(9)

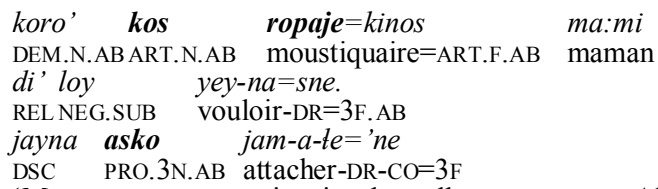

b) PRO correspond à l'argument interne

Pour mettre en relief le participant haut dans la hiérarchie de saillance, normalement exprimé comme argument interne, d'une façon comparable à celle décrite ci-dessus, une opération détransitivisante doit prendre place. Après cette opération, le participant est représenté par PRO en position initiale, suivi par la particule kwey; l'insertion de cette particule rend la construction intransitive, c'est-à-dire, qu'il n'y a plus d'argument interne (voir Haude 2009) ; l'ancien argument externe est optionnellement exprimé comme oblique. La construction transitive et son équivalent détransitivisé sont illustrés en (11).

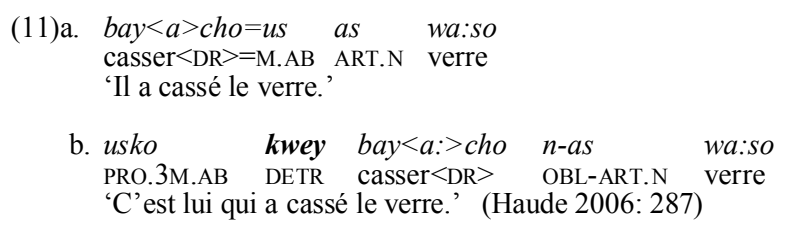

La fonction de la construction avec kwey est pareille à l'expression de l'argument externe en position initiale (a). Typiquement, le participant exprimé I par le pronom a été mentionné juste avant, comme dans les exemples suivants :

7 Un possesseur est codé de la même façon qu'un argument interne, c'est-à-dire par cliticisation interne (voir Haude 2010). La traduction littérale de la première proposition serait « Il y a une moustiquaire de maman ». 
(12) seys is pul-a-cho:-pa

six ART.PL balayer-DR-CLF.INTERIEUR-AGT

i'ko kwey nokowa resibir-na no-kos,

PRO.3PL DETR FUT recevoir-DR OBL-ART.N.AB

eney, sotak-poy di' wa:ka

REMPL un-CL.animal REL vache

'Il y a six balayeuses (de l'église). Elles recevront maintenant, eh, une vache (de l'église) (et pas les autres qui réclament une vache aussi).'

(13) kinos ney senyo:ra, Roxana Suárez

ART.F.AB ici madame Roxana Suárez

isne kwey joy-a:-te n-isne

PRO.3F.AB DETR aller-DR-CO OBL-PRO.F.AB

'Cette dame, Roxana Suárez, elle l'a emmenée.'

(14) i'ne, i:ni. I'ne kwey way-na

PRO.3F DEM.3F.PRX PRO.3F DETR prendre-DR

n-os jotkwa

OBL-ART.N.PSE œuf

'Elle, celle-ci. C'est elle qui a pris l'œuf.' (la petite-fille qui avait volé le dernier œuf de la poule)

Dans le corpus de textes, cette construction se trouve seulement avec un prédicat direct (tandis qu'elle est grammaticale avec un prédicat inverse aussi, voir Haude 2010), autrement dit, c'est toujours l'agent à saillance inhérente qui est mis en relief de cette façon.

Bien qu'il soit obligatoire d'utiliser la construction détransitivisante pour mettre en saillance un participant haut dans la hiérarchie quand il a été introduit immédiatement auparavant, il n'est pas impossible d'exprimer ce participant comme PRO initial sans modification du noyau. Cependant, la fonction de cette dernière construction est assez différente: elle reprend un participant qui avait été un topique auparavant (en (15), le grand-père ; en (16), le fils ; en (17), le mari de la locutrice), tandis que les propositions immédiatement précédentes avaient un autre participant comme topique. La construction est moins emphatique que les constructions de mise en saillance présentées ci-dessus, et ne peut normalement pas être traduite par une construction clivée.

(15) joy-chet is chot komersyante di' juyeni,

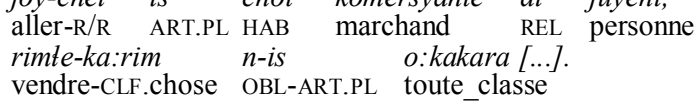

vendre-CLF.chose OBL-ART.PL toute classe

che usko jayna eney ji:sa-na=us os

et PRO.M.AB DSC REMPL faire-DR=3M.AB ART.N.PSE

nego:siyo $n$-is juyeni.

commerce OBL-ART.PL personne

'Les marchands venaient toujours, (ils) vendaient tout, et lui (mon grandpère), euh, il faisait du commerce avec les gens.' 
(16) $\mathrm{Ja}^{\prime}$ to:mi n-is loto:ba $t$ ya:lo:we, seulement eau OBL-ART.PL cruche 1.INTR boire ban usko rimet-na=us.

mais PRO.M.AB acheter-DR $=3 \mathrm{M}$. AB

'Ce n'est que l'eau de la cruche que je bois (je ne bois que de l'eau de la cruche), mais lui (mon fils) achète (les boissons).'

(17) jayna ney kwey no-kos ima:yoj jayna jo'yaj--isne.

DSC ici IM OBL-ART.N.AB matin DSC arriver--3F.AB

u'ko invitar-na=u--k-isne bo os

PRO.M inviter-DR=3M--OBV-3F.AB pour ART.N.PSE

jiwa-wa=sne n-as des'ayuno-wa=sne ney.

venir-NML $=3 \mathrm{~F} . \mathrm{AB} \quad$ OBL-ART. $\mathrm{N}$ petit-déjeûner-NML=3F.AB ici

'Aujourd'hui dans la matinée, elle (absente) arriva, lui (mon mari, présent)

l'avait invitée pour qu'elle vienne prendre le petit-déjeûner ici.'

\section{SAILLANCE DISCURSIVE : PREMIERE ET DEUXIEME PERSONNES}

Comme nous l'avons vu dans la section 3, dans le noyau d'une proposition transitive, les personnes locales, en accord avec leur saillance inhérente, ne peuvent être exprimées que comme argument interne. Pour mettre en relief une personne locale, on s'attend donc à l'opération détransitivisante (construction b) qui permet que le participant exprimé comme l'argument interne soit représenté par PRO. Pourtant, la construction a) - qui contient un PRO initial et un argument interne non-coréférentiel - est possible aussi avec les personnes locales, même quand la personne mise en relief est supérieure dans la hiérarchie. Dans les paragraphes suivants, nous présentons premièrement la construction attendue dans le contexte local - laquelle avec l'opération détransitivisante, (b) - , et après, la moins attendue - sans la détransitivisation (a).

La construction détransitivisée avec des personnes locales est illustrée dans (18)-(21). Elle n'est pas fréquente avec les pronoms de $1^{\mathrm{e}}$ et $2^{\mathrm{e}}$ personnes, et le corpus de textes ne comporte que des exemples à la $1^{\mathrm{e}}$ personne ; les exemples avec la $2^{\mathrm{e}}$ personne (20)-(21) sont sollicités. Dans tous les cas, et à la différence de la construction à troisième personne vue plus haut, la construction semble impliquer un sens de focus contrastif, indiquant que ce n'est aucune autre personne qui réalise l'action; cette différence peut être due au fait que la première personne est la plus haute dans la hiérarchie de saillance. De la même façon qu'avec les $3^{\text {es }}$ personnes, c'est seulement avec un prédicat direct qu'on trouve cette construction.

(18) kas rey baw $<a>r a-w a=n$ kos pasaje $=n$

NEG MOD payer $<$ DR $>$-NML $=2$ ART.N.AB billet $=2$

bo inta, inta kwey baw<a: $>r a$

parce.que PRO.1SG PRO.1SG DETR payer $<$ DR $>$

'Tu ne paieras pas ton billet, parce que c'est moi, c'est moi qui vais le payer.'

(19) iy'li kwey way-na ni-kinos majniw- $a=n$

1 PL DETR prendre-DR OBL-ART.F.AB enfant_de-EP=2 
'C'est nous qui avons pris ta fille (quand elle était perdue).'

(20)

ulkwat nokowa kwey il-na n-as bebetkwa

PRO.2SG maintenant DETR étendre-DR $=2$ OBL-ART.N cuir

'C'est toi qui vas étendre le cuir maintenant.' [soll]

(21) iy'bikwet kwey sal-na no-kos mora:ni

PRO.2PL DETR chercher-DR OBL-ART.N.AB manquer

'C'est vous qui allez chercher ce qui manque.' [soll]

La construction où PRO est coréférentiel avec l'argument interne, illustrée en | (15)-(17) ci-dessus pour les $3^{\text {es }}$ personnes, est très fréquente avec les personnes locales. Par contraste avec les $3^{\text {es }}$ personnes, avec des personnes locales cette construction semble avoir une fonction de focus contrastif aussi, la différence avec la construction détransitivisée n'étant pas claire. Egalement aux autres cas décrits ici, il n'y a pas de pause entre le pronom libre et le reste de la phrase.

L'exemple (22) montre la première et (23) la deuxième personne exprimées comme PRO (la présence du marqueur zéro pour la première personne (22) est indiquée par l'accentuation du verbe : l'accent reste sur l'avant-dernière syllabe ; voir Haude 2011).

(22) ban inta ona-sinet-na=0--is

mais PRO.1SG connaître-CLF.langue-DR=1SG--3PL.AB

'(Ils ne me comprenaient pas,) mais moi, je les comprenais.'

(23) jayna kas ten $<a>$ pante:-wa $=\varnothing$ as joy-wa $=\varnothing$,

DSC NEG pouvoir $<$ DR $>-\mathrm{NML}=1 \mathrm{SG}$ ART.N aller-NML $=1 \mathrm{SG}$

ban ulkwat jayna ona-waj-na=n

mais PRO.2SG DSC connaître-CL.lieu-DR=2SG

'Je ne peux plus y aller, mais toi, tu connais déjà le lieu.'

Nous discuterons maintenant la mise en relief d'une personne locale sans l'opération détransitivisante, où PRO correspond à l'argument externe.

Comme nous l'avons vu plus haut, dans un scénario intralocutif (interaction entre $1^{\mathrm{e}}$ et $2^{\mathrm{e}}$ personnes), seule la $1^{\mathrm{e}}$ personne est exprimée dans le noyau de la proposition transitive. La $2^{\mathrm{e}}$ personne est inférée du contexte ou, sinon, elle peut être spécifiée par un pronom initial, comme l'illustrent (24) et (25), qui représentent la construction a).

(24) ulkwat kay-a:-poj=Ø

2SG manger-DR-CAUS $=1 \mathrm{SG}$

'Je te donne à manger.' [soll]

(25) ulkwat it lawajes-kay $=\varnothing$ papi:to

PRO.2SG 1 remède-INV $=1 \mathrm{SG}$ papa

'Tu m'as guérie, papa.'

Cette construction ne semble pas toujours avoir une fonction emphatique; elle peut simplement viser à désambiguïser le sens : comme l'argument externe n'est 
pas obligatoirement exprimé, hors contexte il pourrait aussi s'agir d'une troisième personne.

D'une façon assez inattendue au vu des règles décrites en section 3, dans un scénario contenant une personne locale, cette stratégie peut aussi servir à mettre en relief la personne supérieure, bien que cette personne ne puisse jamais être exprimée comme argument externe dans le noyau. Autrement dit, on peut imaginer que dans cette construction, l'argument interne est extrait du noyau syntaxique et mis, sous forme de PRO, devant le prédicat; le prédicat prend alors comme argument interne l'autre participant de l'événement et est marqué comme direct/inverse selon le rôle sémantique de ce dernier. Cette stratégie peut être nommé a'), car formellement elle est pareille à la construction a). Cependant, ici, PRO est [+saillant], et l'argument interne est [-saillant].

Les exemples(26) et (27) montrent ce phénomène pour le scénario local, où la $1^{\mathrm{e}}$ personne est exprimée par un pronom libre, laissant le pronom de $2^{\mathrm{e}}$ personne occuper la position d'argument interne. Comme l'argument interne est le patient, le prédicat est marqué comme inverse - bien que l'action soit effectuée par le participant supérieur dans la hiérarchie. (Les exemples donnés ici sont les seuls de ce type trouvés dans le corpus.)

(26) inta disoy oyloni-kay- $a=n k w e t$

PRO.1SG CNTF accompagner-INV-EP=2PL

'Moi, je vous aurais accompagnés (même si j'avais été le seul à le faire).'

(27) inta jempitet chot loj-'oj-et-kay-a=n

PRO.1SG toujours HAB laver-CL.vêtements-APPL-INV-EP=2

'C'est moi qui te fais toujours ta lessive.' [soll]

Cette construction est aussi possible quand une personne locale interagit avec une $3^{\mathrm{e}}$ personne. Dans les exemples suivants (les seuls trouvés jusqu'ici), la $1^{\mathrm{e}}$ personne patient est exprimée comme PRO. En (28) et (29), les deux participants sont des êtres humains ; en revanche, en (30), où le pronom en position initiale est celui de la première personne inclusive ('toi et moi'), l'agent est un animal.

inta naychi-poj-na=us

PRO.1SG premier-CAUS-DR $=3 \mathrm{M} . \mathrm{AB}$

'Il me fit marcher en tête, moi (au lieu de marcher en tête lui-même).'

(29) ka:, ulkwat yey-na='ne

\section{non PRO.2SG vouloir-DR $=3 \mathrm{~F}$}

'Non, c'est toi qu'elle veut (pour t'écouter parler movima)!'

$$
\begin{aligned}
& \text { jayna kas ve vel-wa=a is pa:ko } \\
& \text { DSC NEG DR regarder-NML=3N ART.PL chien } \\
& \text { bo jayna i:de, i:de sal-na=a } \\
& \text { parce.que DSC PRO.1INCL PRO.1 INCL chercher-DR=3N } \\
& \text { '(Quand un jaguar s'aperçoit qu'il y a des hommes à proximité), il ne regarde } \\
& \text { plus les chiens, parce que c'est nous, c'est nous qu'il cherche.' }
\end{aligned}
$$


Cette construction étant rare, la différence entre elle et la construction détransitivisante (b) n'est pas facile à définir. L'apparition de la construction a') au lieu de b) pourrait s'expliquer par la forme du verbe (direct ou inverse). Dans les exemples (28)-(29), le verbe est direct, et la personne mise en relief est donc le patient. Comme nous l'avons constaté plus haut, la construction détransitivisante b) n'est pas utilisée avec la forme inverse du verbe. L'exemple suivant, sollicité, montre que la construction a') est préférée pour mettre en relief un patient local, tandis que la construction b) n'est pas acceptée. ${ }^{8}$

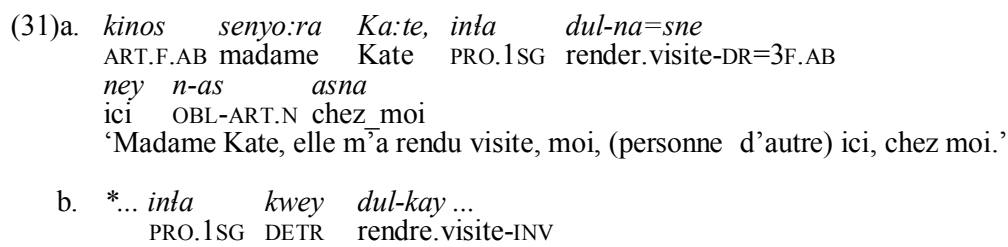

Quant à la construction inverse en (26), le verbe oylonikay 'être accompagné par' (du verbe intransitif oyloni 'accompagner') n'est pas attesté en forme directe, et pourrait donc être exclue de la construction détransitivisante ${ }^{9}$; cette question mérite une recherche approfondie. Nous n'avons aucune explication pour l'utilisation de la forme inverse en (27) (au lieu de la forme directe dans la construction détransitivisante), qui provient d'une sollicitation et doit peut-être être revue.

\section{CONCLUSION}

Dans cet article nous avons discuté la structure de la proposition transitive en movima, qui est fondée sur la saillance inhérente des référents nominaux, et une construction destinée à mettre en relief un participant du discours. Dans une proposition transitive simple, l'argument correspondant au participant supérieur en termes de saillance inhérente, comme la $1^{\mathrm{e}}$ ou $2^{\mathrm{e}}$ personne ou une $3^{\mathrm{e}}$ personne connue d'après le contexte, est exprimé comme argument interne (faisant partie du syntagme prédicatif), et l'argument correspondant au participant inférieur en termes de saillance inhérente, par exemple une $3^{\mathrm{e}}$ personne nouvelle dans le discours, est exprimé comme argument externe au syntagme prédicatif, de ce fait omissible. La saillance inhérente de la $1^{\mathrm{e}}$ et $2^{\mathrm{e}}$ personnes est grammaticalement fixe : ces personnes ne peuvent être exprimées que comme argument interne. En revanche, dans le cas où deux $3^{\text {es }}$ personnes interagissent, le choix est largement déterminé par leur topicalité. En général on peut dire que plus le référent est haut en termes de saillance inhérente, moins il est marqué en termes de matériel

${ }^{8}$ La locutrice l'a jugé non-grammatical, mais les données sont vacillantes_sur ce point. 9 En général, les bases se terminant en -ni ne peuvent jamais être marquées par le direct, la suffixation du marqueur -na créant un nom locatif. 
phonologique : un argument interne est généralement un pronom - la première personne au singulier est même signalée par un zéro - tandis qu'un argument externe est souvent un syntagme nominal, qui peut être relativisé (comme dans l'exemple (9)).

Tout participant peut être mis en relief pour des fins discursives par un pronom libre (PRO) au début de la proposition. Lorsque PRO correspond à l'argument externe, cela n'a pas d'effet sur la structure du noyau (à ceci près qu'en général, l'argument externe n'est pas additionnellement exprimé dans le noyau). La fonction de cette construction est une reprise d'un participant introduit juste auparavant. Pour mettre en relief le participant normalement codé comme argument interne, donc celui qui est haut dans la hiérarchie de saillance, la situation change : il est nécessaire d'employer une opération détransitivisante pour obtenir le même effet qu'avec l'argument externe. Si ce participant est exprimé comme pronom initial sans aucune modification du noyau - qui inclut toujours l'argument interne, coréférentiel avec le pronom initial -, ce n'est pas une mise en relief très prononcée, mais une reprise d'un participant qui était un topique plus tôt dans le discours.

La mise en saillance d'un référent bas dans la hiérarchie de saillance est donc plus simple que celle d'un participant haut dans la hiérarchie. Du point de vue de la structure informationnelle, cela est compréhensible, car une entité haute dans la hiérarchie a plus d'affinité avec le topique, c'est-à-dire, le participant donné ou présupposé, tandis que l'expression par PRO est plutôt liée à la focalisation. Ainsi la grammaire movima réflète une opposition entre la saillance inhérente, reliée à la topicalité, et la saillance discursive, reliée à la focalisation.

Quand il s'agit de mettre en relief une $1^{\mathrm{e}}$ ou $2^{\mathrm{e}}$ personne, qui ne peuvent être exprimées que comme argument interne, il y a une possibilité supplémentaire (rare en termes de fréquence) : ici, même la personne supérieure peut être focalisée par un pronom initial, avec pour effet que la personne inférieure peut occuper la position d'argument interne. En termes de structure, cela montre que la position du pronom initial est en dehors du noyau syntaxique; en termes de fonction, cela montre que même une personne à haute saillance inhérente peut être traitée comme un participant inférieur. La stratégie de mise en saillance discursive peut donc neutraliser la base qui détermine la structure de la proposition transitive, c'est-à-dire la saillance inhérente.

Il faut souligner que cette étude, ayant pris en compte surtout des propositions isolées ou avec très peu de contexte, ne peut être que le début d'une recherche plus approfondie qui examine l'expression des participants dans des segments plus larges du discours.

\section{SYMBOLES ET ABREVIATIONS:}

$\sim$ = réduplication; $\langle>=$ infixe; -- = cliticisation externe; $\mathrm{AB}=$ absent; $\mathrm{APPL}=$ applicatif; $\mathrm{ART}=$ article; $\mathrm{CAUS}=$ causatif; $\mathrm{CL}=$ classificateur; $\mathrm{CNTF}=$ contrefactuel; $\quad \mathrm{CO}=$ co-participant $\quad$ (applicatif); $\mathrm{DEM}=$ démonstratif; DETR $=$ détransitivisant; $\quad \mathrm{DR}=$ direct; $\quad \mathrm{DSC}=$ discontinuatif; $\quad$ DUR=duratif; $\mathrm{EP}=$ epenthétique; EXCL=exclamatif; $\mathrm{EV}=$ évidentiel; $\mathrm{F}=$ féminin; FUT=futur; 
$\mathrm{HAB}=$ habituel; $\mathrm{HYP}=$ hypothétique; INTR=intransitif; INV=inverse; $\mathrm{M}=$ masculin; $\mathrm{MOD}=$ modal; $\mathrm{N}=$ neutre; $\quad \mathrm{NEG}=$ négation; $\mathrm{NML}=$ nominaliseur; $\mathrm{OBL}=\mathrm{oblique}$ $\mathrm{OBV}=$ obviatif; $\mathrm{PL}=$ pluriel; $\mathrm{PRO}=$ pronom; $\mathrm{PRO}=$ pronom libre; $\mathrm{PRX}=$ proche de l'énonciateur; $\quad \mathrm{PSE}=$ passé; $\mathrm{R} / \mathrm{R}=$ réfléchi/réciproque; $\mathrm{REL}=$ relativiseur; $\mathrm{REMPL}=$ remplisseur; $\mathrm{SG}=$ singulier

\section{RÉFÉRENCES BIBLIOGRAPHIQUES}

Comrie B., 1989, Language Universals and Linguistic Typology: Syntax and Morphology, Chicago, Chicago University Press, $2^{\mathrm{e}}$ édition.

Croft W., 2003, Typology and Universals, Cambridge, Cambridge University Press, 2 édition.

DuBois J. A., 1987, The discourse basis of ergativity, Language 63-4, p. 805-855.

Haude K., 2006, A Grammar of Movima, thèse de doctorat, Nimègue.

Haude K., 2009, Hierarchical alignment in Movima, International Journal of American Linguistics 75-4, p. 513-532.

Haude K., 2010, The intransitive basis of Movima clause structure, in: Gildea, S. et F. Queixalós (eds.), Ergativity in Amazonia, Amsterdam/Philadelphia, Benjamins, p. 285-315.

Haude K., 2011, Argument encoding in Movima: the local domain, International Journal of American Linguistics 77-4, numéro spécial sur Argument Encoding Systems in the Languages of Lowland Bolivia, Rose \& Guillaume (eds), p. 559-575.

Klaiman M., 1991, Grammatical Voice, Cambridge, Cambridge University Press. 